\title{
Real-world survival outcomes with immune checkpoint inhibitors in large- cell neuroendocrine tumors of lung
}

\author{
Elizabeth Dudnik (D) ,, ${ }^{1,2}$ Samuel Kareff, ${ }^{3}$ Mor Moskovitz, ${ }^{4}$ Chul Kim (D) , ${ }^{3}$ \\ Stephen V Liu (D) , ${ }^{3}$ Anastasiya Lobachov, ${ }^{5}$ Teodor Gottfried, ${ }^{5}$ Damien Urban, ${ }^{5}$ \\ Alona Zer, ${ }^{1,2}$ Ofer Rotem, ${ }^{1}$ Amir Onn, ${ }^{2,6}$ Mira Wollner, ${ }^{4}$ Jair Bar, ${ }^{2,5}$ Israel Lung Cancer \\ Group
}

To cite: Dudnik E, Kareff S, Moskovitz M, et al. Realworld survival outcomes with immune checkpoint inhibitors in large-cell neuroendocrine tumors of lung. Journal for ImmunoTherapy of Cancer 2021;9:e001999. doi:10.1136/ jitc-2020-001999

ED and SK contributed equally. Accepted 29 December 2020

\section{Check for updates}

(C) Author(s) (or their employer(s)) 2021. Re-use permitted under CC BY-NC. No commercial re-use. See rights and permissions. Published by BMJ.

${ }^{1}$ Thoracic Cancer Service, Davidoff Cancer Center, Rabin Medical Center, Beilinson Campus, Petah Tikva, Israel ${ }^{2}$ Sackler Faculty of Medicine, Tel Aviv University, Tel Aviv, Israel

${ }^{3}$ Lombardi Comprehensive Cancer Center, MedStar

Georgetown University Hospital, Washington, District of

Columbia, USA

${ }^{4}$ Thoracic Cancer Service, Rambam Health Care Campus, Haifa, Israel

${ }^{5}$ Institute of Oncology, Sheba Medical Center, Tel HaShomer, Ramat Gan, Israel

${ }^{6}$ Pulmonology Institute, Sheba Medical Center, Tel Hashomer, Ramat Gan, Israel

\section{Correspondence to} Dr Elizabeth Dudnik; elizabeth.dudnik1603@gmail. com

\section{ABSTRACT}

Background Little is known regarding the efficacy of immune checkpoint inhibitors (ICI) in patients with advanced large-cell neuroendocrine lung carcinoma (aLCNEC).

Methods 125 consecutive patients with aLCNEC were identified in the electronic databases of 4 participating cancer centers. The patients were divided into group $A$ (patients who received $\mathrm{ICI}, \mathrm{n}=41$ ) and group $B$ (patients who did not receive $\mathrm{ICI}, \mathrm{n}=84$ ). Overall survival since advanced disease diagnosis (OS DX) and OS since ICI initiation (OS ICl) were captured.

Results With a median follow-up of 11.8 months (mo) (IQR 7.5-17.9) and 6.0mo (IQR 3.1-10.9), 66\% and 76\% of patients died in groups $A$ and $B$, respectively. Median OS DX was $12.4 \mathrm{mo}(95 \% \mathrm{Cl} 10.7$ to 23.4$)$ and $6.0 \mathrm{mo}(95 \% \mathrm{Cl}$ 4.7 to 9.4 ) in groups $A$ and $B$, respectively (log-rank test, $\mathrm{p}=0.02)$. For ICl administration, HR for OS DX was 0.59 ( $95 \% \mathrm{Cl} 0.38$ to $0.93, p=0.02$-unadjusted), and 0.58 (95\% Cl 0.34 to $0.98, p=0.04$-adjusted for age, Eastern Cooperative Oncology Group (ECOG) performance status (PS), presence of liver metastases and chemotherapy administration). In a propensity score matching analysis $(n=74 ; 37$ patients in each group matched for age and ECOG PS), median OS DX was $12.5 \mathrm{mo}$ (95\% Cl 10.6 to $25.2)$ and $8.4 \mathrm{mo}(95 \% \mathrm{Cl} 5.4$ to 16.9$)$ in matched groups $A$ and $B$, respectively (log-rank test, $p=0.046)$. OS ICI for patients receiving $\mathrm{ICl}$ as monotherapy $(\mathrm{n}=36)$ was $11.0 \mathrm{mo}$ (95\% Cl 6.1 to 19.4).

Conclusions With the limitations of retrospective design and small sample size, the results of this real-world cohort analysis suggest a positive impact of ICI on OS in aLCNEC.

\section{BACKGROUND}

Large-cell neuroendocrine carcinoma of lung (LCNEC) is a rare malignancy $(<3 \%$ of all lung tumors) characterized by aggressive behavior and high recurrence rate. ${ }^{1-3}$ According to 2015 WHO criteria, LCNEC is composed of large cells with neuroendocrine differentiation (as demonstrated by typical morphology and immunoreactivity for chromogranin, synaptophysin or CD56), and, typically, a high mitotic rate. ${ }^{4}$ In up to
$20 \%$ of cases, LCNEC demonstrates a mixed histology with an additional non-small cell lung cancer (NSCLC) component. ${ }^{4}$ From a molecular perspective, LCNEC can be further classified as SCLC-like subtype (characterized by tumor protein p53 (TP53) and Retinoblastoma gene-1 (RB1) comutations or loss) or NSCLC-like subtype (characterized by lack of $T P 53+R B 1$ alterations and presence of mutations in the Kirsten rat sarcoma viral oncogene homolog, serine/threonine kinase 11, and Kelch-like ECH-associated protein 1 genes); LCNEC molecular subtype may affect response to systemic therapy. ${ }^{5-7}$

Systemic approach in advanced-stage LCNEC primarily includes platinum-based chemotherapy, with objective response rate (ORR) in the range of $12 \%-52 \%$, median progression-free survival (mPFS) of 4.6-6.1 months and median overall survival (OS) of 10.2-11.1 months. ${ }^{28-10}$ The roles of somatostatin analogs and tyrosine-kinase inhibitors (TKIs) in the management of advanced LCNEC remain limited. ${ }^{289}$

Anti-programmed cell death-1 (antiPD-1)/antiprogrammed cell death ligand-1 (anti-PD-L1) immune checkpoint inhibitors (ICI) are well incorporated into treatment algorithms for advanced NSCLC and SCLC-either with or without platinumbased chemotherapy. This is based on the results of numerous randomized clinical trials demonstrating a consistent OS benefit with early incorporation of immunotherapy across histological tumor types. ${ }^{11-20}$ The data regarding clinical activity of ICI in advanced LCNEC, while encouraging, is limited to case reports and small retrospective series. ${ }^{21-31}$ According to the results of several retrospective analyzes, ICI administration in advanced LCNEC is associated with ORR of $13 \%-60 \%$, mPFS of $4.2-14.2$ months and 
median OS of 11.8 months. ${ }^{29-31}$ For instance, the nonrandomized DART trial assessed the clinical activity of combination nivolumab and ipilimumab in rare tumor types and reported an objective response in two out of three patients with advanced LCNEC. ${ }^{32}$ The impact of ICI on OS in advanced LCNEC, however, has not been fully determined. The largest report of ICI in patients with LCNEC from the American National Cancer Database, delivered in abstract form only, included only 37 patients. It did, however, suggest a positive impact of ICI on OS. ${ }^{33}$

Aiming to bridge this gap in the literature, we conducted a retrospective analysis of consecutive patients with advanced LCNEC treated at four tertiary cancer centers. We explored the impact of ICI on OS in this rare lung tumor type, with a keen emphasis on molecular subtype.

\section{MATERIALS AND METHODS}

\section{Patient selection and group assignment}

Electronic databases of four participating cancer centers (Institute of Oncology, Sheba Medical Center, Tel HaShomer; Davidoff Cancer Center, Rabin Medical Center, Beilinson Campus; Rambam Healthcare Campus (Israel); Lombardi Comprehensive Cancer Center, MedStar Georgetown University Hospital (USA)) were systematically searched for patients diagnosed with LCNEC between 2009 and 2019. Mixed LCNEC and small-cell neuroendocrine tumors (NET) of lung origin as well as mixed LCNEC and non-small-cell lung carcinomas with a predominating LCNEC component were also included. Only advanced-stage tumors (stage IV or stage III disease not amenable to definitive treatment, or recurrent tumors not amenable to definitive treatment) were selected. Cases with Ki- $67<30 \%$ were excluded from the analysis given that, in small biopsies, this commonly accepted cut-off is frequently used to separate high-grade LCNEC and SCLC from low-grade NET (typical and atypical carcinoid) in the presence of crush artifact and poor tissue preservation. ${ }^{44-38}$

The patients were then divided into group A (patients who received ICI as any treatment line) and group B (patients who did not receive ICI whatsoever). Group $\mathrm{A}^{*}$ subcategorized patients receiving ICI administered as monotherapy or in combination with different ICI agents, thereby excluding patients who received combination ICI and chemotherapy. Additionally, patients with an available molecular tumor profile were further classified into SCLC-like subtype (defined by the presence of TP53/RB1 co-mutations or loss) or NSCLC-like subtype (defined by the lack of $T P 53 / R B 1$ alterations).

\section{Study design and assessments}

After obtaining institutional ethical review board approval, we retrospectively reviewed patients' charts and hospital electronic medical records, and gathered baseline demographic, clinical, pathological and treatment characteristics. OS since advanced disease diagnosis (OS DX) was captured and compared between groups A and $\mathrm{B}$ among the whole cohort (primary endpoint) and between selected subgroups (according to age, Eastern Cooperative Oncology Group performance status (ECOG PS), presence of liver metastases and tumor molecular subtype). A propensity score matching analysis of OS DX was done, and patients in both groups were matched for age and ECOG PS. Univariate and multivariate analyzes were then performed to assess the impact of patient baseline characteristics, tumor subtype and treatment characteristics on OS DX. Additionally, OS after ICI initiation (OS ICI) was analyzed in group $\mathrm{A}^{*}$ according to the tumor molecular subtype, with an additional univariate analysis exploring the impact of patient baseline characteristics, tumor subtype and treatment characteristics.

OS DX was defined as the time from advanced disease diagnosis until death or censored at last follow-up. OS ICI was calculated from the time of ICI initiation until death or censored at last follow-up. Duration of follow-up was calculated from the time of advanced disease diagnosis until last follow-up or censored at death. The cut-off date for data collection was January 132020.

\section{Statistical analysis}

The sample size was determined by the available patients meeting the inclusion criteria. We conducted the statistical analysis using $\mathrm{R}$ Core Team software ( $\mathrm{R}$ Foundation for Statistical Computing, Vienna, Austria), version $2019 .^{39}$ Categorical variables were presented by numbers and percentiles. Continuous variables were reported by medians and ranges. Categorical variables were compared using the $\chi^{2}$ method or Fisher's exact test, while continuous variables were compared using either a t-test or Mann-Whitney-Wilcoxon test. OS was assessed by the Kaplan-Meier method, with the log-rank test for comparison between groups. The propensity score matching analysis matching patients in the two compared groups for age and ECOG PS was performed with a caliper of 0.5 , a 1:1 matching (ICI administration vs no ICI administration), and an AUC (area under the curve) of 0.67. Cox proportional hazards univariable models including prespecified covariates were constructed. Covariates for the multivariate Cox regression model were selected from the statistically significant covariates found in the aforementioned univariate model. $P$ values less than 0.05 were considered statistically significant. No correction for multiple comparisons was performed.

\section{RESULTS \\ Patient and tumor characteristics}

One hundred eighty-four consecutive patients with histologically confirmed LCNEC diagnosed between 2009 and 2019 were identified from the four participating cancer centers. Forty-nine patients with early-stage disease were excluded from the analysis, and an additional ten cases with Ki-67 $<30 \%$ were excluded as well. The selected cohort thus comprised 125 patients (Thoracic Oncology Service, Institute of Oncology, Sheba Medical Center, Tel 
HaShomer, n=53; Davidoff Cancer Center, Rabin Medical Center, Beilinson Campus, $\mathrm{n}=47$; Thoracic Cancer Service, Rambam Healthcare Campus, n=14; Lombardi Comprehensive Cancer Center, MedStar Georgetown University Hospital, n=11; online supplemental figure S1).

Of the selected 125 patients, 41 were treated with ICI (group A), and 84 did not receive ICI (group B). Baseline demographic, clinical and pathological characteristics for these 125 included are displayed in table 1 . This cohort mainly comprised smokers (84\%); the majority were males $(62 \%)$. Patients with ECOG PS 2-4 at the time of advanced disease diagnosis constituted $26 \%$ of the cohort; brain metastases were present in $32 \%$, and liver metastasis in $34 \%$ of the patients. Patients in group A demonstrated a younger median age of 63 years (IQR 58-68) compared with group B median age of 67.5 years (IQR 62-75) $(\mathrm{p}=0.003)$. Group A included more patients with ECOG PS 0 or $1(75 \%)$ compared with group B $(44 \%)(\mathrm{p}=0.02)$.

Given these differences in baseline characteristics, we performed a propensity score matching analysis to account for age and ECOG PS. The matched cohort $(\mathrm{n}=74)$ included 37 patients in each group with a median age of 64 years for both groups (IQR 59-68 and 59-69 in matched groups $\mathrm{A}$ and $\mathrm{B}$, respectively) $(\mathrm{p}=0.79)$; the proportion of matched patients with ECOG PS 0 or 1 yielded $84 \%$ and $78 \%$ in matched groups $\mathrm{A}$ and $\mathrm{B}$, respectively $(\mathrm{p}=0.77)$, while the percentage of matched patients with ECOG PS 2-4 was 16\% and 22\% in matched groups A and B, respectively $(\mathrm{p}=0.77)$. No other significant differences in baseline patient and tumor characteristics between the matched groups were observed (online supplemental table S1).

Use of molecular tumor testing was generally limited. Comprehensive genomic profiling was complete for only 16 patients (39\%) and 6 patients $(7 \%)$ in groups $A$ and $B$, respectively. PD-L1 expression was assessed in 21 patients $(51 \%)$ and 14 patients $(17 \%)$ in groups $\mathrm{A}$ and $\mathrm{B}$, respectively. Tumor mutation burden was available in seven patients $(17 \%)$ and one patient $(1 \%)$ in groups $\mathrm{A}$ and $\mathrm{B}$, respectively. Finally, microsatellite instability was calculated in eight patients (19\%) in group A only (table 1).

Group A* comprised 36 patients. This group's baseline characteristics according to tumor molecular subtype are presented in online supplemental table S2. Since the molecular testing was likewise limited (39\% of patients in group $\left.A^{*}, n=14\right)$, tumors with NSCLC-like molecular subtype $(n=9)$ were compared with all others $(n=27$, including tumors with SCLC-like subtype $(n=5)$ and tumors with unknown molecular subtype $(n=22))$. In group $\mathrm{A}^{*}$, NSCLC-like tumors were more likely to have a mixed histology $(\mathrm{p}=0.03)$.

\section{Treatment characteristics}

ICI regimens used in group A included nivolumab $(\mathrm{n}=19,46 \%$ of patients in group A), pembrolizumab $(\mathrm{n}=4,10 \%)$, atezolizumab $(\mathrm{n}=6,15 \%)$, durvalumab $(\mathrm{n}=3$, $7 \%)$, nivolumab/ipilimumab $(\mathrm{n}=4,10 \%)$, platinum/ pemetrexed/pembrolizumab $(\mathrm{n}=3,7 \%)$ and platinum/ etoposide/atezolizumab $(n=2,5 \%)$.

Significantly more patients in group A $(95 \%)$ compared with group B $(74 \%)$ received chemotherapy $(p=0.01)$ (table 1). The proportion of patients receiving SCLCbased chemotherapy regimens (eg, cisplatin/etoposide and carboplatin/etoposide) was numerically similar $(73 \%$ and $61 \%$ in groups A and B, respectively) $(\mathrm{p}=0.40)$. Thirty-six percent of patients in group A and $20 \%$ of patients in group B received NSCLC-based chemotherapy regimens $(\mathrm{p}=0.11)$; such NSCLC-based regimens included platinum/pemetrexed $(n=9)$, platinum/paclitaxel $(n=6)$, platinum/pemetrexed/bevacizumab ( $\mathrm{n}=4)$, platinum/ vinorelbine $(n=4)$, pemetrexed $(n=3)$, paclitaxel $(n=2)$, docetaxel $(n=2)$, vinorelbine $(n=2)$, platinum/docetaxel $(n=1)$, gemcitabine $(n=1)$, gemcitabine/paclitaxel $(n=1)$, and capecitabine/temozolomide $(n=1)$. Three patients in group B received somatostatin analogs. Two patients in group B and one patient in group A received epidermal growth factor receptor (EGFR) TKIs on diagnosis of an activating mutation in the EGFR gene (exon $19 \mathrm{del}$ ); two additional patients in group A received anaplastic lymphoma kinaseTKIs, though confirmatory comprehensive tumor molecular testing did not confirm the presence of a targetable abnormality, later halting such treatment. Overall, patients in group A received more lines of systemic treatment $(\mathrm{p}<0.001)$.

In the matched cohort $(\mathrm{n}=74)$, no significant differences between groups were observed in terms of chemotherapy administration or chemotherapy regimens (online supplemental table S1).

Treatment characteristics of patients in group $\mathrm{A}^{*}$ are presented in the online supplemental table S2. Systemic treatments were similar between patients with NSCLClike molecular tumor subtype and the rest of the group. Most patients received nivolumab, pembrolizumab or atezolizumab as second-line treatment.

\section{OS since advanced disease diagnosis}

After a median follow-up period of 11.8 months (IQR 7.5-17.9) for group A and 6.0 months (IQR 3.1-10.9) for group B, $(\mathrm{p}<0.001$ for the comparison), 27 (66\%) patients died in group A while $64(76 \%)$ patients died in group B. Median OS DX was 12.4 months (95\% CI 10.7 to 23.4) in group A and 6.0 months (95\% CI 4.7 to 9.4) in group $\mathrm{B}(\mathrm{p}=0.02)$ (figure $1 \mathrm{~A})$. In group $\mathrm{A}$, projected 1-year and 2-year survival rates since advanced disease diagnosis were $55 \%$ and $25 \%$, respectively (figure $1 \mathrm{~A}$ ). In group B, projected 1-year and 2-year survival rates since advanced disease diagnosis were $32 \%$ and $18 \%$, respectively (figure 1A).

In the matched cohort $(\mathrm{n}=74)$, median follow-up comprised 12.0 months (IQR 6.5-19.9) for matched group A and 6.1 months (IQR 4.4-10.1) for matched group $\mathrm{B}, \quad(\mathrm{p}=0.004)$. Twenty-four $(65 \%)$ patients in matched group A and $26(70 \%)$ patients in matched group B died during the study period. Median OS DX was 12.5 months (95\% CI 10.6 to 25.2) in matched group 
Table 1 Baseline clinical, pathological and treatment characteristics of patients with advanced LCNEC divided according to exposure to $\mathrm{ICl}$

\begin{tabular}{|c|c|c|c|c|}
\hline & $\begin{array}{l}\text { Pts treated with ICI } \\
\text { (group } A, n=41 \text { ) }\end{array}$ & $\begin{array}{l}\text { Pts not treated with ICI } \\
\text { (group } B, n=84 \text { ) }\end{array}$ & $P$ value & All pts $(n=125$ \\
\hline Age, years-median (IQR) & $63(58-68)$ & $67(62-75)$ & 0.003 & $66(61-73)$ \\
\hline Sex, n (\%) & & & 1.00 & \\
\hline Female & $16(39)$ & $32(38)$ & & $48(38)$ \\
\hline Male & $25(61)$ & $52(62)$ & & $77(62)$ \\
\hline Smoking history, $\mathrm{n}(\%)$ & & & 1.00 & \\
\hline Current/past smoker & $36(88)$ & $69(82)$ & & $105(84)$ \\
\hline Never smoker & $5(12)$ & $11(13)$ & & $16(13)$ \\
\hline NA & $0(0)$ & $4(5)$ & & $4(3)$ \\
\hline Histological subtype, n (\%) & & & 0.12 & \\
\hline LCNEC & $33(80)$ & $73(87)$ & & $106(85)$ \\
\hline Mixed LCNEC+SCLC & $6(15)$ & $11(13)$ & & $17(14)$ \\
\hline Mixed LCNEC+NSCLC & $2(5)$ & $0(0)$ & & $2(1)$ \\
\hline Stage, $\mathrm{n}(\%)^{*}$ & & & 0.77 & \\
\hline $1 / I I$ & $4(10)$ & $11(13)$ & & $15(12)$ \\
\hline III/IV & $37(90)$ & $71(85)$ & & $108(87)$ \\
\hline NA & $0(0)$ & $2(2)$ & & $2(1)$ \\
\hline ECOG PS, n (\%) & & & 0.02 & \\
\hline $0 / 1$ & $31(75)$ & $37(44)$ & & $68(54)$ \\
\hline $2 / 3 / 4$ & $6(15)$ & $26(31)$ & & $32(26)$ \\
\hline NA & $4(10)$ & $21(25)$ & & $25(20)$ \\
\hline Brain metastases, $\mathrm{n}(\%)$ & & & 0.97 & \\
\hline Yes & $14(34)$ & $26(31)$ & & $40(32)$ \\
\hline No & $27(66)$ & $46(55)$ & & $73(58)$ \\
\hline NA & $0(0)$ & $12(14)$ & & $12(10)$ \\
\hline Liver metastases, $\mathrm{n}(\%)$ & & & 0.80 & \\
\hline Yes & $15(37)$ & $27(32)$ & & $42(34)$ \\
\hline No & $26(63)$ & $56(67)$ & & $82(65)$ \\
\hline NA & $0(0)$ & $1(1)$ & & $1(1)$ \\
\hline Ki-67, \% - median (IQR)† & $70(60-80)$ & $70(50-80)$ & 0.39 & $70(50-80)$ \\
\hline Molecular subtype & & & NA & \\
\hline SCLC-like & $6(15)$ & $1(1)$ & & $7(6)$ \\
\hline NSCLC-like & $10(24)$ & $5(6)$ & & $15(12)$ \\
\hline NA & $25(61)$ & $78(93)$ & & $103(82)$ \\
\hline PD-L1 TPS, n (\%) & & & 0.15 & \\
\hline$\geq 50 \%$ & $3(7)$ & $0(0)$ & & $3(2)$ \\
\hline $1 \%-49 \%$ & $2(5)$ & $4(5)$ & & $6(5)$ \\
\hline$<1 \%$ & $16(39)$ & $10(12)$ & & $26(21)$ \\
\hline NA & $20(49)$ & $70(83)$ & & $90(72)$ \\
\hline TMB, mut/Mb-median (IQR) $\ddagger$ & $14(10-17)$ & $21(21)$ & 0.38 & $14(10-21)$ \\
\hline MSI-high, n (\%)§ & $1(2)$ & NA & NA & $1(1)$ \\
\hline \multicolumn{5}{|l|}{ Treatment details } \\
\hline Chemotherapy, n (\%) & $39(95)$ & $62(74)$ & 0.01 & $101(81)$ \\
\hline SCLC-based chemotherapy, $\mathrm{n}(\%) \emptyset$ & $30(73)$ & $51(61)$ & 0.40 & $81(65)$ \\
\hline
\end{tabular}


Table 1 Continued

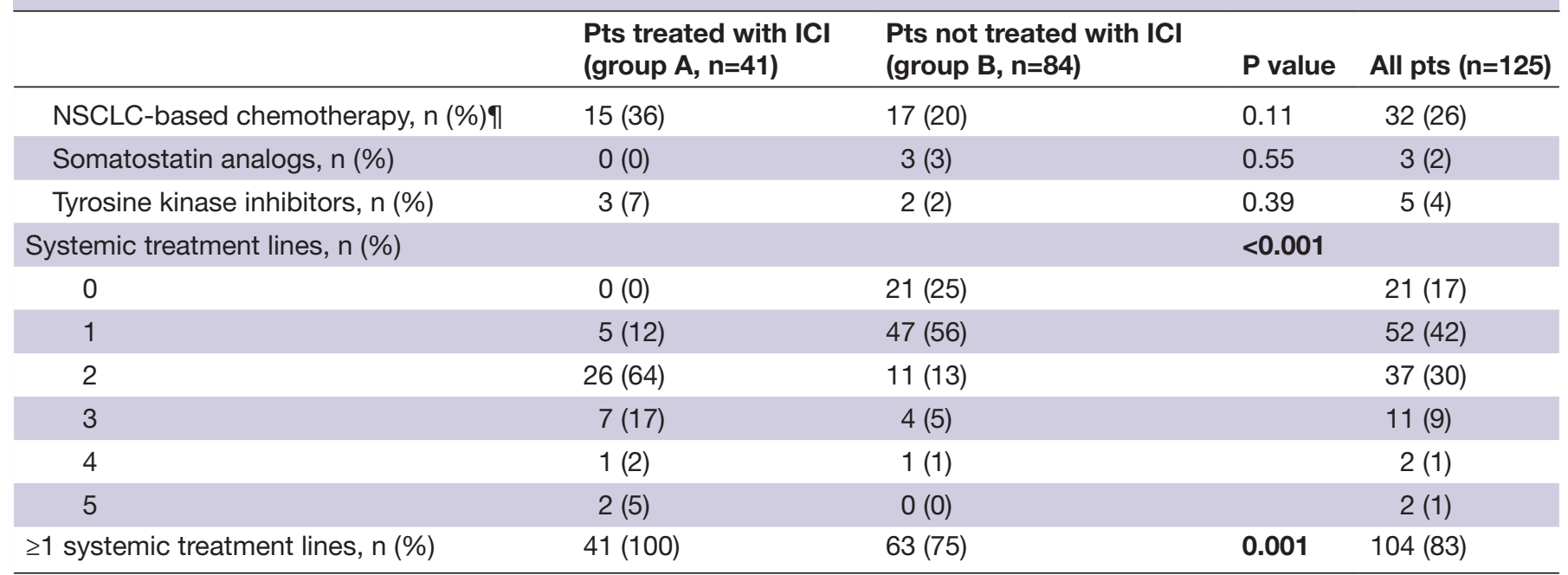

Statistically significant differences are indicated in bold.

*Stage at initial diagnosis.

†Assessed in 88 patients (group $A, n=33$; group $B, n=55$ ).

$\ddagger$ Assessed in eight pts (group $A, n=7$; group $B, n=1$ ).

$\S$ Assessed in eight pts (group $A, n=8$ ).

ף14 patients ( $n=6$ and $n=8 \%-15 \%$ and $10 \%$ of groups $A$ and $B$, respectively) received both SCLC-based and NSCLC-based chemotherapy. ECOG PS, Eastern Cooperative Oncology Group performance status score; ICl, immune check point inhibitors; LCNEC, large-cell neuroendocrine tumors of lung; MSI, microsatellite instability; mut/Mb, mutations per megabase; NA, not available/not applicable; NSCLC, non-small-cell lung cancer; PD-L1, programmed-death ligand 1; pts, patients; SCLC, small-cell lung cancer; TMB, tumor mutation burden; TPS, Tumor Proportion Score.

A and 8.4 months (95\% CI 5.4 to 16.9$)$ in matched group B $(p=0.046)$ (figure 1B). In matched group A, projected 1-year and 2-year survival rates since advanced disease diagnosis were $57 \%$ and $27 \%$, respectively (figure $1 \mathrm{~B}$ ). For matched group B, projected 1-year and 2-year survival rates since advanced disease diagnosis were $33 \%$ and $19 \%$, respectively (figure $1 \mathrm{~B}$ ).

\section{Univariate and multivariate analysis of OS DX}

In the univariate analysis, age $(p=0.02)$, ECOG PS on diagnosis of advanced disease $(\mathrm{p}<0.001)$, presence of liver metastases $(p=0.005)$, chemotherapy administration $(\mathrm{p}<0.001)$ and ICI administration $(\mathrm{p}=0.02)$ all

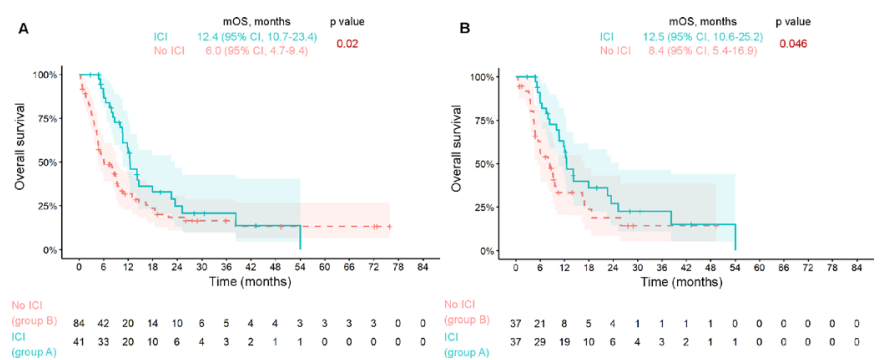

Figure 1 OS of patients with advanced LCNEC according to $\mathrm{ICl}$ exposure in the entire cohort $(A, n=125)$, and in the cohort matched for age and ECOG PS (B, n-74): group Apatients who received $\mathrm{ICl}$; group $\mathrm{B}-$ patients who did not receive ICI. ECOG PS, Eastern Cooperative Oncology Group performance status score; $\mathrm{ICl}$, immune check point inhibitors; LCNEC, large-cell neuroendocrine tumors of lung; mOS, median overall survival. demonstrated a significant correlation with OS DX, whereas sex, smoking status, molecular subtype, stage at diagnosis and presence of brain metastases did not ( $>00.05)$ (table 2).

ICI administration $(\mathrm{p}=0.04)$, chemotherapy administration ( $p=0.002)$, ECOG-PS on diagnosis of advanced disease $(\mathrm{p}=0.002)$ and presence of liver metastasis $(p=0.03)$ remained statistically associated with OS DX in a multivariate Cox regression analysis model that incorporated all factors found to significantly correlate with OS DX in univariate analysis (table 2).

\section{OS DX in selected subgroups}

We analyzed the effect of ICI exposure on OS DX in several patient subgroups (figure 2). ICI administration positively affected OS DX in elderly ( $\geq 65$ years old) patients $(\mathrm{p}=0.03)$ and patients without liver metastases $(\mathrm{p}=0.05)$. A trend toward longer OS DX with ICI exposure was seen in patients with ECOG PS 0 or $1(\mathrm{p}=0.052)$. In smaller subgroups of patients younger than 65 years $(\mathrm{p}=0.45)$, patients with liver metastases $(\mathrm{p}=0.09)$, and patients with ECOG PS 2-4 ( $\mathrm{p}=0.2)$, there was no statistically significant OS benefit seen with ICI administration. Additionally, the smaller subgroup of patients with NSCLC-like tumors did not seem to derive OS benefit from ICI administration $(\mathrm{p}=0.63)$ as opposed to the remainder of the cohort comprizing patients with SCLClike or unknown molecular subtype tumors $(\mathrm{p}=0.02)$ (figure 2). 
Table 2 Univariate and multivariate COX regression analyzes of overall survival since diagnosis of advanced disease in patients with advanced LCNEC

\begin{tabular}{|c|c|c|c|c|}
\hline \multirow[b]{2}{*}{ Parameters } & \multicolumn{2}{|l|}{ Univariate analysis } & \multicolumn{2}{|c|}{ Multivariate analysis } \\
\hline & HR $(95 \% \mathrm{Cl})$ & $P$ value & HR (95\% Cl) & $P$ value \\
\hline $\mathrm{ICl}$ : yes versus no & 0.59 (0.38 to 0.93$)$ & 0.02 & 0.58 (0.34 to 0.98$)$ & 0.04 \\
\hline Chemotherapy: yes versus no & 0.33 (0.20 to 0.52$)$ & $<0.001$ & 0.41 (0.23 to 0.73$)$ & 0.002 \\
\hline Age & 1.15 (1.02 to 1.29$)$ & 0.02 & 1.00 (0.97 to 1.03$)$ & 0.89 \\
\hline Sex: male versus female & $1.23(0.81$ to 1.88$)$ & 0.34 & & \\
\hline Smoking: yes versus no & $1.00(0.55$ to 1.81$)$ & 1.00 & & \\
\hline Stage at DX:I/II vs III/IV & 1.56 (0.86 to 2.81$)$ & 0.15 & & \\
\hline ECOG PS:2-4 vs 0/1 & 2.66 (1.65 to 4.31$)$ & $<0.001$ & 2.3 (1.37 to 3.84$)$ & 0.002 \\
\hline Brain metastases: yes versus no & 1.40 (0.89 to 2.20$)$ & 0.15 & & \\
\hline Liver metastases: yes versus no & 1.83 (1.19 to 2.80$)$ & 0.005 & 1.70 (1.06 to 2.74$)$ & 0.03 \\
\hline Molecular subtype: NSCLC-like vs others & $0.56(0.28$ to 1.11$)$ & 0.10 & & \\
\hline
\end{tabular}

Statistically significant differences are indicated in bold.

DX, diagnosis; ECOG PS, Eastern Cooperative Oncology Group performance status score; ICl, immune check-point inhibitors; LCNEC, largecell neuroendocrine tumors of lung; NSCLC, non-small-cell lung cancer.

\section{OS with ICI}

After a median follow-up after ICI initiation in group $\mathrm{A}^{*}$ of 6.2 months (IQR 2.7-14.0), $24(67 \%)$ patients died. In group $\mathrm{A}^{*}$, median OS ICI was 11.0 months $(95 \%$ CI 6.1 to 19.4) (figure 3). The projected 1-year and 2-year survival rates after ICI initiation were $44 \%$ and $22 \%$, respectively (figure 3).

Median follow-up after ICI initiation was 6.9 months (IQR 6.1-12.9) in patients with NSCLC-like tumors and 5.7 months (IQR 2.0-14.3) in the remainder of patients in group $A^{*}(p=0.25)$. Six patients $(67 \%$ of patients with NSCLC-like tumor subtype) and 18 patients (67\% of the rest of group $\mathrm{A}^{*}$ ) died. Median OS ICI was 9.3 months (95\% CI 6.1 to not reached (NR)) in patients with NSCLC-like tumors, and 11.0 months (95\% CI 3.7 to NR) in the rest of group $A^{*}(p=0.65)$ (online supplemental figure S2).

In the univariate analysis, only ECOG PS at ICI initiation $(p=0.02)$ and presence of liver metastases $(p=0.01)$ demonstrated a significant correlation with OS ICI. Sex, age, smoking status, stage at diagnosis, PD-L1 TPS $(\geq 1 \%$ vs $<1 \%$ ), molecular subtype (NSCLC-like vs all others), presence of brain metastases, ICI type (monotherapy with an anti-PD-1/PD-L1 agent vs combination of an antiPD-1 agent with an anticytotoxic T-lymphocyte-associated protein 4 (anti-CTLA4) agent), administration of chemotherapy, and number of systemic treatment lines prior to ICI administration did not correlate with OS ICI (online supplemental table S3). Multivariate analysis of OS ICI was not performed because of small sample size.

\section{DISCUSSION}

To the best of our knowledge, our data set represents the largest series to date reporting on mature outcomes of ICI in advanced-stage LCNEC. It is also one of the first analyzes to assess the impact of ICI administration on OS in advanced LCNEC. We found ICI administration in LCNEC to be associated with longer OS DX in the entire cohort, as well as in the cohort matched for age and ECOG PS. The positive impact of ICI administration on OS of patients with advanced LCNEC was further supported by the results of univariate and multivariate analyzes. Since prospective randomized clinical trials focusing on patients with this rare tumor subtype are challenging to complete, these data provide valuable insight regarding possible therapeutic options for advanced LCNEC.

According to our observations, the median OS was twice as long in patients who received ICI (12.4 months, $95 \%$ CI -10.7 to 23.4 ) compared with those who did not (6.0 months, $95 \%$ CI -4.7 to 9.4$)(p=0.02)$. Similar results were detected when matching patients for age and ECOG PS: the median OS was longer in patients who received ICI (12.5 months, 95\% CI -10.6 to 25.2) compared with those who did not (8.4 months, 95\% CI -5.4 to 16.9 ) $(\mathrm{p}=0.046)$. Projected landmark OS rates were also higher in patients who were exposed to ICI: 1-year survival rates of $55 \%$ vs $32 \%-33 \%$, and 2-year survival rates of $25 \%-27 \%$ vs $18 \%-19 \%$ in patients who did and did not receive ICI, respectively. Our observations correspond to the results of the retrospective analysis of advanced LCNEC patients presented by Komiya and Powell as an ASCO 2020 virtual meeting abstract. Analysis of Komiya and Powell demonstrated that the use of ICI was associated with improved OS $(p=0.0168)$; a landmark OS analysis in the ICI group showed 12-month and 18-month survival rates of $34 \%$ and $29 \%$, respectively, compared with $24 \%$ and $15 \%$ in the non-ICI group. ${ }^{33}$

Importantly, the OS rates in patients not exposed to ICI in our cohort were consistent with historical data retrieved from the majority of large retrospective series 
Open access

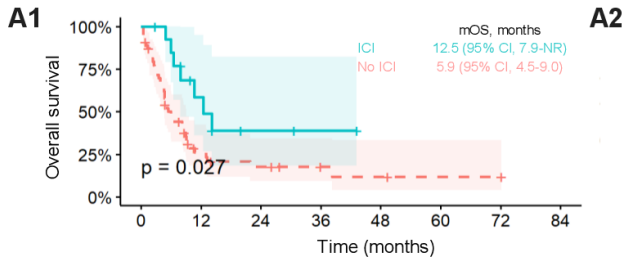

Number at risk

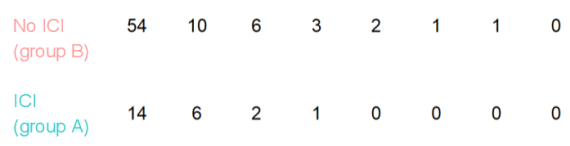

BI

Number at risk

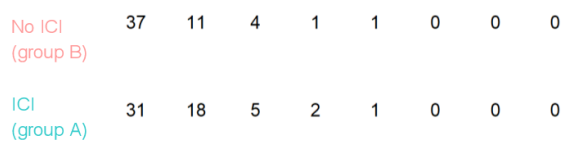

CI

BI

C2

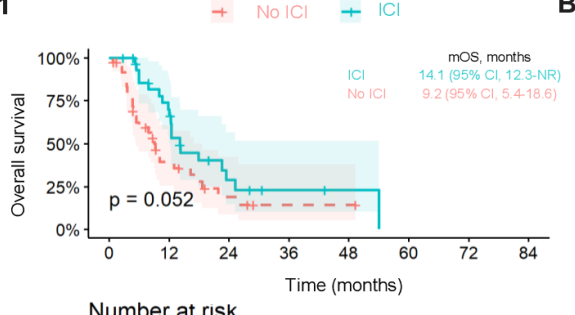

A2

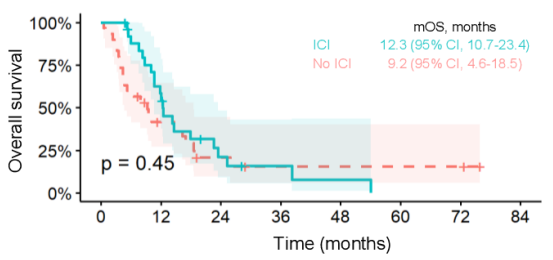

Number at risk
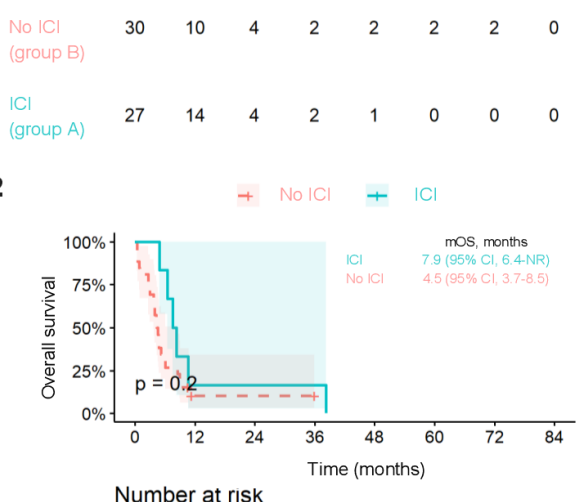

Number at risk
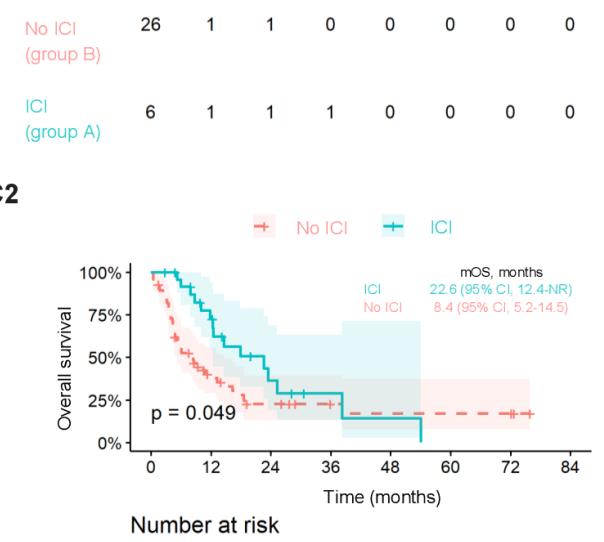

Number at risk
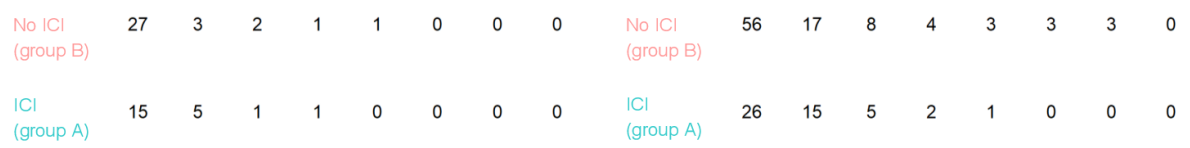

D2

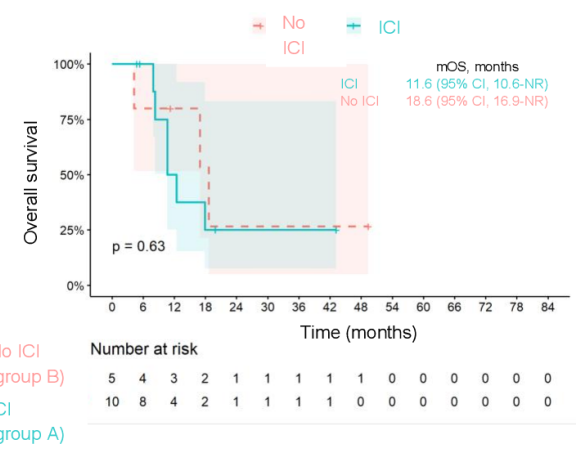

Figure 2 The effect of ICJ exposure on OS of patients with advanced LCNEC in selected subgroups according to age (A1 $\geq 65$ years; A2 $\leq 65$ years), ECOG PS (B1-ECOG PS 0/1; B2-ECOG PS 2-4), liver metastases (C1-liver metastases present; C2-liver metastases absent) and molecular subtype (D1-SCLC-like subtype or unknown molecular subtype; D2-NSCLC-like subtype). ECOG PS, Eastern Cooperative Oncology Group performance status score; ICJ, immune checkpoint inhibitors; LCNEC, largecell neuroendocrine tumors of lung; mOS, median overall survival; NR, not reached; NSCLC, non-small-cell lung cancer; SCLC, small-cell lung cancer.

Dudnik E, et al. J Immunother Cancer 2021;9:e001999. doi:10.1136/jitc-2020-001999

7 


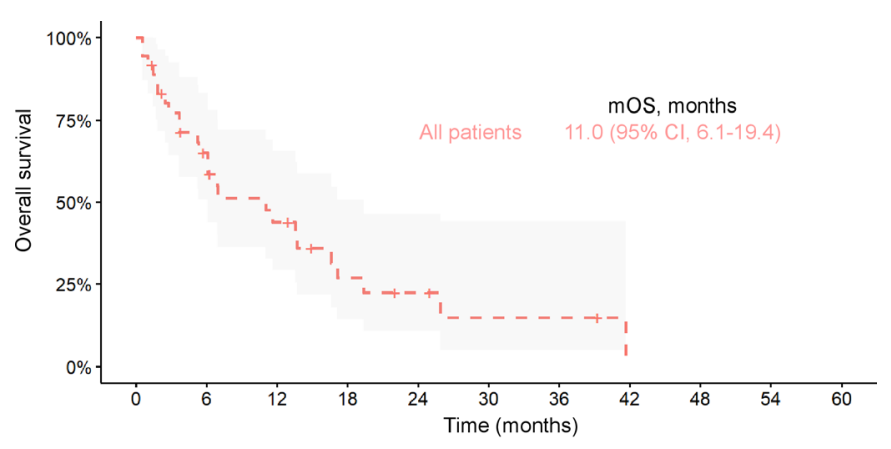

Number at risk

All $\begin{array}{lllllllllll}36 & 20 & 12 & 6 & 4 & 2 & 2 & 0 & 0 & 0 & 0\end{array}$

Figure 3 OS with $\mathrm{ICl}$ in patients with advanced LCNEC.

$\mathrm{ICI}$, immune checkpoint inhibitors; LCNEC, large-cell

neuroendocrine tumors of lung; mOS, median overall survival.

and some prospective clinical studies assessing platinumbased chemotherapy in advanced LCNEC. ${ }^{134041}$ Other prospective studies which evaluated platinum-based chemotherapy in patients with advanced LCNEC reported higher OS rates compared with those we observed, probably reflecting the differences in baseline and treatment characteristics between patients in real-world cohorts and those enrolled in clinical trials. ${ }^{42}$ Our cohort notably included patients with poor prognosis, $25 \%$ of whom did not receive any systemic treatment.

We acknowledge some differences in baseline and treatment characteristics in favor of patients treated with ICI in our presented cohort. Specifically, these patients were younger, had a better ECOG PS at the time of diagnosis of advanced disease, and were more likely to be treated with systemic therapy. However, the results of propensity score matching analysis along with multivariate analysis accounting for these imbalances confirmed a positive correlation between ICI administration and OS beyond traits including younger age, better ECOG PS, lack of liver metastases and administration of chemotherapy. Our conclusions are further supported by the analysis presented by Komiya and Powell which also demonstrated a significant correlation between ICI administration and OS in advanced LCNEC patients, along with chemotherapy administration, surgery, female sex and absence of liver metastases. ${ }^{33}$

We also confirmed our previous observation regarding outcomes related to IO exposure in advanced LCNEC. ${ }^{31}$ In this expanded cohort comprised 36 patients treated with ICI administered as either anti-PD-1/PD-L1 monotherapy or in combination with anti-CTLA 4 therapy, we again observed a median OS of 11.0 months $(95 \%$ CI 6.1 to 19.4 ) - similar to the median OS of 11.8 months (95\% CI 3.7 to NR) we previously had demonstrated in the cohort of 21 patients. Our data set is the only known report to date detailing mature OS outcomes related to ICI exposure in patients with advanced LCNEC.

Of note, no significant correlation was seen between the level of PD-L1 expression and OS with ICI exposure, although the small number of cases with PD-L1 TPS available for analysis limited the value of this observation. Positive
PD-L1 expression in LCNEC represents a rare event, and its prognostic value is controversial. ${ }^{43-46}$ It is unknown if PD-L1 TPS may serve as a predictive factor in the context of ICI therapy in advanced LCNEC, and it remains to be seen whether such a relationship exists with either of the two LCNEC molecular subtypes. We hypothesize such an association might be limited to NSCLC-like LCNEC if one extrapolates known data from NSCLC and SCLC. ${ }^{19} 4748$

One of the major limitations of our study is lack of comprehensive molecular tumor profiling data available for most patients, thereby weakening conclusions regarding the correlation between the established molecular LCNEC subtypes and outcomes related to ICI exposure. Based on data available from our series and another series from Sabari et $a l^{29}$ the NSCLC-like LCNEC subtype appears to derive less benefit from ICI compared with the SCLC-like molecular subtype. For example, the Sabari et al series demonstrated ORR with ICI of $43 \%(3 / 7)$ in SCLC-like LCNEC vs $13 \%(1 / 8)$ in NSCLC-like LCNEC. ${ }^{29}$ In our series, patients with the NSCLC-like molecular subtype trended towards a numerically lower median OS with ICI (9.3 months) compared with the remainder of the patients treated with ICI (11.0 months) $(\mathrm{p}=0.65)$. Additionally, a numerically lower median OS DX was witnessed in NSCLC-like LCNEC patients exposed to ICI (11.6 months) compared with NSCLC-like LCNEC patients not exposed to ICI (18.6 months) ( $\mathrm{p}=0.63$ ), while the opposite held true in the rest of the cohort (ie, 12.5 months and 6.0 months for patients who received and did not receive ICI, respectively) $(\mathrm{p}=0.02)$. Given the overall low molecular testing rate in our cohort, these observations are only hypothesis generating.

Our analysis has additional important limitations, including retrospective nature, lack of central pathological assessment and relatively small sample size of patients treated with ICI.

Prospective phase II clinical trials are underway to assess the efficacy of anti-PD-1/PD-L1 ICI and the combination of anti-PD-1 ICI with anti-CTLA ICI in high-grade neuroendocrine tumors. ${ }^{49}$ Some of these allow enrollment of patients with LCNEC (g, NCT03352934, NCT03190213, NCT03136055, NCT03290079 and NCT02834013). Additional questions remaining to be addressed include the correlation between ICI treatment efficacy and the LCNEC molecular subtype, as well as the value of a combined approach implementing concurrent platinumbased chemotherapy with ICI administration.

In conclusion, the results of this real-world data analysis suggest that the use of ICI to be associated with superior OS in advanced LCNEC. The impact of molecular tumor subtype on ICI outcomes requires further evaluation.

\section{Twitter Chul Kim @chulkimMD and Stephen V Liu @StephenVLiu}

Contributors ED, SK and JB have made substantial contribution to the conception and design of the work, acquisition and analysis of the data; all the listed authors contributed substantially to the data collection and manuscript drafting; and all the listed authors revised the submitted manuscript and approved its final version before submission. 
Funding The authors have not declared a specific grant for this research from any funding agency in the public, commercial or not-for-profit sectors.

Competing interests ED reported consulting fees from MSD, BMS, Astra Zeneca, Roche, Boehringer Ingelheim, Pfizer, Novartis, Takeda. MM reported consulting fees from Boehringer Ingelheim, Roche, Astra Zeneca, MSD, BMS, Abbvie, Takeda, Pomicell. CK reported research funding (to institution) from AstraZeneca, BMS, Novartis, Regeneron, Tesaro, Karyopharm, Debiopharm, Jassen, and Mirati and consulting fees from Novartis. SVL reported consulting fees from AstraZeneca, Beigene, Blueprint, Boehringer Ingelheim, Bristol-Myers Squibb, Catalyst, Celgene, Clovis, Corvus, G1 Therapeutics, Genentech, Guardant Health, Inivata, Janssen, Jazz, Lilly, LOXO, MSD, PharmaMar, Pfizer, Regeneron, and Takeda and research grants (to institution) from Alkermes, AstraZeneca, Bayer, Blueprint, BristolMyers Squibb, Genentech, Lilly, Lycera, Merck, Molecular Partners, Pfizer, Rain Therapeutics, RAPT, Spectrum, and Turning Point Therapeutics. DU reported consulting fees and non-financial support from BMS, Astra Zeneca, Takeda, consulting fees MSD, Roche and Boehringer Ingelheim. AZ reported grants from BMS, personal fees from Roche, MSD, BMS, Astra Zeneca, Takeda. A0 reported advisory fees from MSD, BMS, Roche, Astra Zeneca, Novartis, Boehringer Ingelheim. MW reported speaker fees and funding (to institution) from Roche, MSD. JB reported grants and personal fees from MSD, Roche, BMS, Abbvie, Pfizer, Novartis, Astra Zeneca, Boehringer Ingelheim, Takeda.

Patient consent for publication Not required.

Ethics approval The study was conducted in accordance with the principles of good clinical practice, and institutional review board approval was obtained at each participating oncological center before the study initiation. No patient identifying data were included in the central data collection.

Provenance and peer review Not commissioned; externally peer reviewed.

Data availability statement Data are available on reasonable request. Data are not publicly available according to the Rabin Medical Center's strict institutional policy with regards to public availability of unidentified patient data. However, those data which are minimally required to replicate the outcomes of the study will be made available on reasonable request.

Supplemental material This content has been supplied by the author(s). It has not been vetted by BMJ Publishing Group Limited (BMJ) and may not have been peer-reviewed. Any opinions or recommendations discussed are solely those of the author(s) and are not endorsed by BMJ. BMJ disclaims all liability and responsibility arising from any reliance placed on the content. Where the content includes any translated material, BMJ does not warrant the accuracy and reliability of the translations (including but not limited to local regulations, clinical guidelines, terminology, drug names and drug dosages), and is not responsible for any error and/or omissions arising from translation and adaptation or otherwise.

Open access This is an open access article distributed in accordance with the Creative Commons Attribution Non Commercial (CC BY-NC 4.0) license, which permits others to distribute, remix, adapt, build upon this work non-commercially, and license their derivative works on different terms, provided the original work is properly cited, appropriate credit is given, any changes made indicated, and the use is non-commercial. See http://creativecommons.org/licenses/by-nc/4.0/.

\section{ORCID iDs}

Elizabeth Dudnik http://orcid.org/0000-0001-6971-3576

Chul Kim http://orcid.org/0000-0003-0191-8684

Stephen V Liu http://orcid.org/0000-0002-4852-3914

\section{REFERENCES}

1 Derks JL, Hendriks LE, Buikhuisen WA, et al. Clinical features of large cell neuroendocrine carcinoma: a population-based overview. Eur Respir J 2016;47:615-24.

2 Lo Russo G, Pusceddu S, Proto C, et al. Treatment of lung large cell neuroendocrine carcinoma. Tumour Biol 2016;37:7047-57.

3 Kinoshita T, Yoshida J, Ishii G, et al. The differences of biological behavior based on the clinicopathological data between resectable large-cell neuroendocrine carcinoma and small-cell lung carcinoma. Clin Lung Cancer 2013;14:535-40.

4 Travis WD, Brambilla E, Nicholson AG, et al. The 2015 World Health organization classification of lung tumors: impact of genetic, clinical and radiologic advances since the 2004 classification. J Thorac Oncol 2015;10:1243-60.

5 Rekhtman N, Pietanza MC, Hellmann MD, et al. Next-generation sequencing of pulmonary large cell neuroendocrine carcinoma reveals small cell carcinoma-like and non-small cell carcinoma-like subsets. Clin Cancer Res 2016;22:3618-29.

6 Derks JL, Leblay N, Thunnissen E, et al. Molecular subtypes of pulmonary large-cell neuroendocrine carcinoma predict chemotherapy treatment outcome. Clin Cancer Res 2018;24:33-42.

7 George J, Walter V, Peifer M, et al. Integrative genomic profiling of large-cell neuroendocrine carcinomas reveals distinct subtypes of high-grade neuroendocrine lung tumors. Nat Commun 2018;9:1048.

8 Rossi G, Cavazza A, Marchioni A, et al. Role of chemotherapy and the receptor tyrosine kinases kit, PDGFRalpha, PDGFRbeta, and Met in large-cell neuroendocrine carcinoma of the lung. J Clin Oncol 2005;23:8774-85.

9 Sun J-M, Ahn M-J, Ahn JS, et al. Chemotherapy for pulmonary large cell neuroendocrine carcinoma: similar to that for small cell lung cancer or non-small cell lung cancer? Lung Cancer 2012;77:365-70.

10 Naidoo J, Santos-Zabala ML, lyriboz T, et al. Large cell neuroendocrine carcinoma of the lung: Clinico-pathologic features, treatment, and outcomes. Clin Lung Cancer 2016;17:e121-9.

11 Brahmer J, Reckamp KL, Baas P, et al. Nivolumab versus docetaxel in advanced squamous-cell non-small-cell lung cancer. $N$ Engl $J$ Med 2015;373:123-35.

12 Borghaei H, Paz-Ares L, Horn L, et al. Nivolumab versus docetaxel in advanced nonsquamous non-small-cell lung cancer. $N$ Engl J Med 2015;373:1627-39.

13 Herbst RS, Baas P, Kim D-W, et al. Pembrolizumab versus docetaxel for previously treated, PD-L1-positive, advanced non-small-cell lung cancer (KEYNOTE-010): a randomised controlled trial. Lancet 2016;387:1540-50.

14 Rittmeyer A, Barlesi F, Waterkamp D, et al. Atezolizumab versus docetaxel in patients with previously treated non-small-cell lung cancer (oak): a phase 3, open-label, multicentre randomised controlled trial. Lancet 2017;389:255-65.

15 Reck M, Rodríguez-Abreu D, Robinson AG, et al. Pembrolizumab versus chemotherapy for PD-L1-positive non-small-cell lung cancer. N Engl J Med 2016;375:1823-33.

16 Gandhi L, Rodríguez-Abreu D, Gadgeel S, et al. Pembrolizumab plus chemotherapy in metastatic non-small-cell lung cancer. $N$ Engl $J$ Med 2018;378:2078-92.

17 Paz-Ares L, Luft A, Vicente D, et al. Pembrolizumab plus chemotherapy for squamous non-small-cell lung cancer. $N$ Engl J Med 2018;379:2040-51.

18 Socinski MA, Jotte RM, Cappuzzo F, et al. Atezolizumab for firstline treatment of metastatic nonsquamous NSCLC. N Engl J Med 2018;378:2288-301.

19 Horn L, Mansfield AS, Szczęsna A, et al. First-line atezolizumab plus chemotherapy in extensive-stage small-cell lung cancer. $N$ Engl J Med 2018;379:2220-9.

20 Paz-Ares L, Dvorkin M, Chen Y, et al. Durvalumab plus platinumetoposide versus platinum-etoposide in first-line treatment of extensive-stage small-cell lung cancer (Caspian): a randomised, controlled, open-label, phase 3 trial. Lancet 2019;394:1929-39.

21 Mauclet C, Duplaquet F, Pirard L, et al. Complete tumor response of a locally advanced lung large-cell neuroendocrine carcinoma after palliative thoracic radiotherapy and immunotherapy with nivolumab. Lung Cancer 2019;128:53-6.

22 Wang VE, Urisman A, Albacker L, et al. Checkpoint inhibitor is active against large cell neuroendocrine carcinoma with high tumor mutation burden. J Immunother Cancer 2017;5:3-6.

23 Chauhan A, Arnold SM, Kolesar J, et al. Immune checkpoint inhibitors in large cell neuroendocrine carcinoma: current status. Oncotarget 2018;9:14738-40.

24 Daido W, Yamasaki M, Saito N, et al. [Effectiveness of Nivolumab in Large-Cell Neuroendocrine Carcinoma of the Lung - A Report of Two Cases]. Gan To Kagaku Ryoho 2017;44:59-62.

25 Zhang X, Sun Y, Miao Y, et al. Immune Checkpoint Inhibitor Therapy Achieved Complete Response for Drug-Sensitive EGFR/ALK Mutation-Negative Metastatic Pulmonary Large-Cell Neuroendocrine Carcinoma with High Tumor Mutation Burden: A Case Report. Onco Targets Ther 2020;13:8245-50.

26 Takimoto Sato M, Ikezawa Y, Sato M, et al. Large cell neuroendocrine carcinoma of the lung that responded to nivolumab: a case report. Mol Clin Oncol 2020;13:43-7.

27 Qin Y, Yu M, Zhou L, et al. Durable response to combination radiotherapy and immunotherapy in EP-resistant lung large-cell neuroendocrine carcinoma with B2M and STK11 mutations: a case report. Immunotherapy 2020;12:223-7.

28 Oda R, Okuda K, Yamashita Y, et al. Long-term survivor of pulmonary combined large cell neuroendocrine carcinoma treated with nivolumab. Thorac Cancer 2020;11:2036-9. 
29 Sabari JK, Julian RA, Ni A, et al. Outcomes of advanced pulmonary large cell neuroendocrine carcinoma stratified by RB1 loss, SLFN11 expression, and tumor mutational burden. JCO 2018;36:e20568.

30 Levra MG, Mazieres J, Valette CA, et al. P1.07-012 efficacy of immune checkpoint inhibitors in large cell neuroendocrine lung cancer: results from a French retrospective cohort. $J$ Thorac Oncol 2017;12:S702-3.

31 Sherman S, Rotem O, Shochat T, et al. Efficacy of immune checkpoint inhibitors (ICPi) in large cell neuroendocrine tumors of lung (LCNEC). Lung Cancer 2020;143:40-6.

32 Patel SP, Othus M, Chae YK, et al. Swog 1609 (dart): a phase II basket trial of dual anti-CTLA-4 and anti-PD-1 blockade in rare tumors. JCO 2019;37:TPS2658.

33 Komiya T, Powell E. Role of immunotherapy in stage IV large cell neuroendocrine carcinoma of the lung. JCO 2020;38:9060.

34 Pelosi G, Rindi G, Travis WD, et al. Ki-67 antigen in lung neuroendocrine tumors: unraveling a role in clinical practice. $J$ Thorac Oncol 2014;9:273-84.

35 Pelosi G, Rodriguez J, Viale G, et al. Typical and atypical pulmonary carcinoid tumor overdiagnosed as small-cell carcinoma on biopsy specimens: a major pitfall in the management of lung cancer patients. Am J Surg Pathol 2005;29:179-87.

36 Aslan DL, Gulbahce HE, Pambuccian SE, et al. Ki-67 immunoreactivity in the differential diagnosis of pulmonary neuroendocrine neoplasms in specimens with extensive crush artifact. Am J Clin Pathol 2005;123:874-8.

37 Lin O, Olgac S, Green I, et al. Immunohistochemical staining of cytologic smears with MIB-1 helps distinguish low-grade from high-grade neuroendocrine neoplasms. Am J Clin Pathol 2003;120:209-16.

38 Helpap B, Köllermann J. Immunohistochemical analysis of the proliferative activity of neuroendocrine tumors from various organs. are there indications for a neuroendocrine tumor-carcinoma sequence? Virchows Arch 2001;438:86-91.
39 R Foundation for Statistical Computing. A language and environment for statistical computing. Vienna, Austria, 2019.

40 Derks JL, van Suylen RJ, Thunnissen E, et al. Chemotherapy for pulmonary large cell neuroendocrine carcinomas: does the regimen matter? Eur Respir J 2017;49:1601838.

41 Le Treut J, Sault MC, Lena H, et al. Multicentre phase II study of cisplatin-etoposide chemotherapy for advanced large-cell neuroendocrine lung carcinoma: the GFPC 0302 study. Ann Oncol 2013;24:1548-52.

42 Niho S, Kenmotsu H, Sekine I, et al. Combination chemotherapy with irinotecan and cisplatin for large-cell neuroendocrine carcinoma of the lung: a multicenter phase II study. J Thorac Oncol 2013;8:980-4.

43 Hermans BCM, Derks JL, Thunnissen E, et al. Prevalence and prognostic value of PD-L1 expression in molecular subtypes of metastatic large cell neuroendocrine carcinoma (LCNEC). Lung Cancer 2019;130:179-86.

44 Tsuruoka K, Horinouchi H, Goto Y, et al. PD-L1 expression in neuroendocrine tumors of the lung. Lung Cancer 2017;108:115-20.

45 Eichhorn F, Harms A, Warth A, et al. PD-L1 expression in large cell neuroendocrine carcinoma of the lung. Lung Cancer 2018;118:76-82.

46 Fan Y, Ma K, Wang C, et al. Prognostic value of PD-L1 and PD-1 expression in pulmonary neuroendocrine tumors. Onco Targets Ther 2016;9:6075-82.

47 Garon EB, Rizvi NA, Hui R, et al. Pembrolizumab for the treatment of non-small-cell lung cancer. N Engl J Med 2015;372:2018-28.

48 Mok TSK, Wu Y-L, Kudaba I, et al. Pembrolizumab versus chemotherapy for previously untreated, PD-L1-expressing, locally advanced or metastatic non-small-cell lung cancer (KEYNOTE-042): a randomised, open-label, controlled, phase 3 trial. Lancet 2019;393:1819-30.

49 Weber MM, Fottner C. Immune checkpoint inhibitors in the treatment of patients with neuroendocrine neoplasia. Oncol Res Treat 2018;41:306-12. 